\title{
Structural characterization of quadruplex DNA with in-cell EPR approaches
}

\author{
Isabelle T. Holder, Malte Drescher, Jörg S. Hartig* \\ Department of Chemistry and Konstanz Research School of Chemical Biology, University of Konstanz, 78467 Konstanz, Germany
}

\section{Keywords:}

Noncanonical nucleic acids

Human telomeric quadruplex

EPR spectroscopy

Site-directed spin-labeling

\begin{abstract}
A B S T R A C T
Guanosine rich DNA sequences have the potential to adopt four stranded conformations termed quadru plexes. The chromosomes of higher organisms are capped by so called telomeres that are composed of repeats of the sequence TTAGGG. Up to 200 nucleotides of the G rich strand form an overhang that is suspected to fold into intramolecular G quadruplexes. Since induction of quadruplexes at the telomeres results in anti proliferative effects, the intracellular structure of $G$ quadruplexes is of high interest as an anti cancer drug target. Here we give a perspective on the elucidation of DNA sequence folds by electron paramagnetic resonance (EPR) distance measurements. The technique complements $\mathrm{X}$ ray crystallography and NMR spectroscopy, as it can be applied in noncrystalline states, is not intrinsically limited by the size of the bio macromolecular complex, and is able to analyze flexible structures or coex isting DNA conformation.
\end{abstract}

\section{Introduction}

Nucleic acids are utilized for information storage by every living organism. Inside the nucleus, DNA usually occurs as a double stranded, right handed helix. ${ }^{1}$ Apart from this well known struc ture, DNA can adopt several noncanonical conformations. ${ }^{2}$ For example branched DNA molecules appear during DNA metabolism processes such as replication, repair or recombination. ${ }^{3-6}$ Holliday junctions ${ }^{7}$ are well described cruciforms, containing four double helical arms that branch from a central junction. More complex DNA structures occur when more than two DNA strands interact with each other. In triple stranded DNA, one strand binds via Hoo gsteen or reverse Hoogsteen hydrogen bonds to the major groove of a B form double helix. Such structures can either be formed in ter or intra molecularly in pyrimidine or purine rich regions. ${ }^{8-10}$ DNA quartet structures, more often called G quadruplexes, are made up of Hoogsteen hydrogen bonded $G$ tetrads that can stack on one another.

Interestingly, most of these structures appear in particular se quence patterns, such as guanosine rich regions. Computational studies showed that $G$ quadruplex motifs are widely distributed in human and bacterial genomes. ${ }^{11-13}$ Whether they form alterna tive DNA structures in vivo is unclear, although their potential to do so was described in vitro by a variety of methods. Quadruplexes often show structural polymorphism characterized by many possi ble conformations, see Section 2 for details. A common approach

\footnotetext{
* Corresponding author. Tel.: +49 7531884575.

E-mail address: joerg.hartig@uni-konstanz.de (J.S. Hartig).
}

used to characterize $\mathrm{G}$ quadruplexes in vitro is circular dichroism (CD) spectroscopy. It is used to distinguish between parallel and anti parallel conformations. ${ }^{14}$ The interpretation of $\mathrm{CD}$ spectra is an empirical approach and multiple species and hybrid structures are difficult to distinguish. For this reason, although a powerful technique, CD spectroscopy data tend to be over interpreted and sometimes have produced controversial results. ${ }^{15} \mathrm{X}$ ray crystallog raphy ${ }^{16}$ and NMR ${ }^{17}$ methodology have been used frequently in or der to obtain full high resolution structures of $G$ quadruplexes. However, the biological relevance of $G$ quadruplex conformations obtained in crystalline states needs to be considered carefully. Growing conditions for crystals may lead to conformational con versions or select nondominant species, especially since alternate quadruplex conformations often possess very similar stabilities. Even NMR spectroscopy has its limitations. Structure determina tion by NMR requires the formation of a kinetically stable species in solution; co existence of multiple species complicates the access of structural information. However, many G quadruplex structures have been characterized by NMR in vitro. ${ }^{17}$ Most of them are thought to form under physiological conditions and to have certain in vivo functions.

To prove their in vivo existence, methods that elucidate DNA structures in cells are necessary. Site directed spin labeling in combination with electron paramagnetic resonance (EPR) is one promising method for probing noncanonical DNA structures. Novel in cell EPR allows for studying DNA topologies in cellulo. In this perspective we focus on EPR measurements of G quadruplex DNA. We first give an overview of quadruplex DNA structures, describing their biochemical characteristics and their potential 
roles in vivo. We depict EPR measurements in combination with site directed spin labeling of DNA and conclude by discussing lim itations and future developments of in cell EPR.

\section{G-quadruplex structures}

G quadruplexes are helical nucleic acid structures that can form within certain guanosine rich sequences. A G quadruplex com prises at least two tetrad layers, each composed of 4 guanines sta bilized by noncanonical Hoogsteen basepairs in a coplanar arrangement (Fig. 1A). ${ }^{18}$ Through $\pi \pi$ interactions, at least two of these tetrads stack upon each other and form a $G$ quartet. The guanosines can be located on one strand, interrupted by loops of different length, but appear on different DNA strands where the Hoogsteen bonds occur intermolecularly. Quadruplexes can adopt a variety of topologies which depend on the orientation of the DNA strands: parallel, antiparallel or hybrid structures are known (Fig. 1C E). The conformation of the glycosidic bond differs depending on topology: it can adopt syn or anti conformation (Fig. 2B). The adopted quadruplex structure and stability are fur ther influenced by the loop length and the sequence composition of the total quadruplex motif. ${ }^{19,20}$ Quadruplexes are stabilized by monovalent cations or small molecule compounds interacting with the $G$ tetrads and thereby neutralizing the electrostatic repulsion of guanine oxygen that point inwards. ${ }^{21-24}$

Several computational studies screened genomic sequences for quadruplex motifs that have four runs of guanines, in which each $\mathrm{G}$ tract is composed of at least 3 guanines. ${ }^{11-13}$ For the human gen ome more than 375,000 motifs have been found. ${ }^{25,26}$ Those studies showed that quadruplex motifs are not randomly distributed in human, yeast and bacterial genomes. They rather appear within certain genomic regions: up and down stream of transcription start sites, near transcription factor binding sites, within the ribo somal DNA, near promoters, in telomeres or within micro and minisatellites. ${ }^{11,12,25-28}$ It also seems that essential guanosines in the quadruplex motifs are more conserved than nucleotides that do not disrupt quadruplex formation of the motif. ${ }^{29}$ These facts suggest that quadruplexes have important functions in genetic processes. $^{2}$ They may function as regulators during transcrip tion, ${ }^{21,30,31}$ replication ${ }^{32-34}$ or recombination. ${ }^{35}$ Some studies gave hints for quadruplex existence in vivo. For different $G$ quadruplex structures, antibodies or fluorescent biomarkers have been devel oped. ${ }^{36-38}$ Recently Balasubramanian and co workers described a structure specific antibody binding to DNA quadruplexes. ${ }^{39}$ They were able to visualize $G$ quadruplexes in the genomic DNA of hu man cells and demonstrated coherence between the cell cycle and quadruplex formation. The visualization of $G$ quadruplexes via fluorescent compounds in cells supports the assumption that those structures actually form in vivo. Nevertheless, although said com pounds show $G$ quadruplex specificity in vitro, the in vivo struc ture of a $G$ quadruplex in genomic DNA remains to be clarified.

\subsection{The human telomeric quadruplex}

Telomere sequences are located at the ends of chromosomes. They comprise a double stranded region with a single stranded $3^{\prime}$ overhang. The whole human telomeric DNA region consists of $5^{\prime} \mathrm{d}$ (TTAGGG) 3' repeats (Fig. 2E) and is typically $58 \mathrm{~kb}$ long with a $3^{\prime}$ end overhang of the $G$ rich strand of up to 200 nucleotides in length. ${ }^{40,41}$ After each somatic cell division the single stranded overhang progressively decreases in length, until the cell under goes apoptosis. ${ }^{42,43}$ The enzyme telomerase, a reverse transcrip tase, can elongate the telomeric ends after cell division. It is usually inactivated in most somatic cells, but highly activated in $8090 \%$ of cancer cells. ${ }^{44}$ Intramolecular and antiparallel quadru plex structures have been shown to block telomerase activity, whereas intermolecular and parallel structures did not. ${ }^{45-47}$ There fore targeting the human telomeric quadruplex (HTQ) is of great research interest for cancer therapeutics. ${ }^{48-50}$ An understanding of the folding of human telomeric $G$ quadruplexes in vivo and their formed structures under physiological conditions will be very ben eficial for a rational based drug design. In addition, the formation of telomeric quadruplexes has been implicated in processes related to aging ${ }^{51,52}$ and genetic stability. ${ }^{53}$ The structural characterization of this sequence is important in order to gain new insights into possible mechanisms.

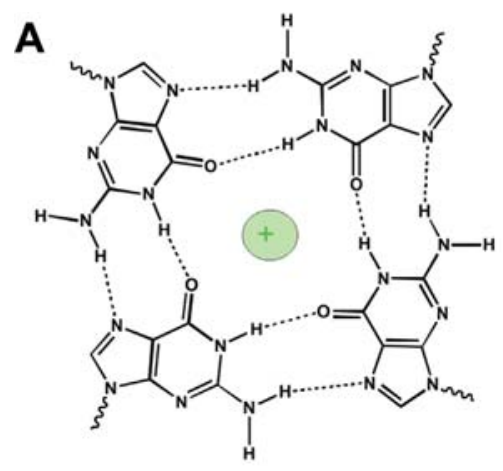

C

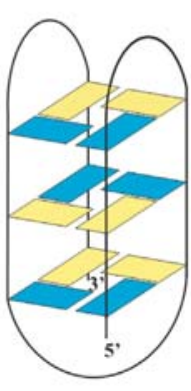

B

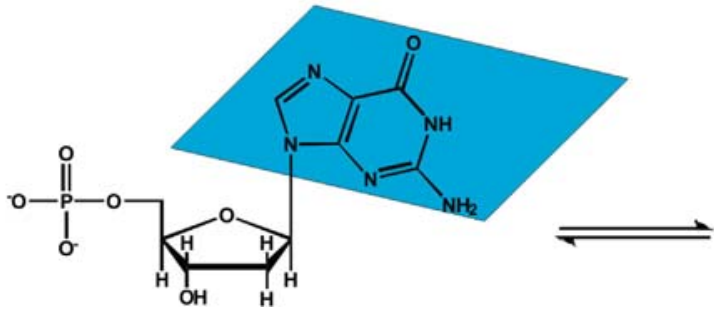

D


Figure 1. Quadruplex structures and topologies. (A) Coplanar arrangement of guanines by noncanonical Hoogsteen base pairing. The tetrad can be stabilized by monovalent cations (especially potassium) that fit into the center. (B) Glycosidic conformations of guanines. Syn-Conformation is depicted yellow and anti is shown in blue. (C-E) different quadruplex topologies, with the respective glycosidic guanines: (C) antiparallel basket type; (D) (3+1) hybrid; (E) parallel propeller type. 
A

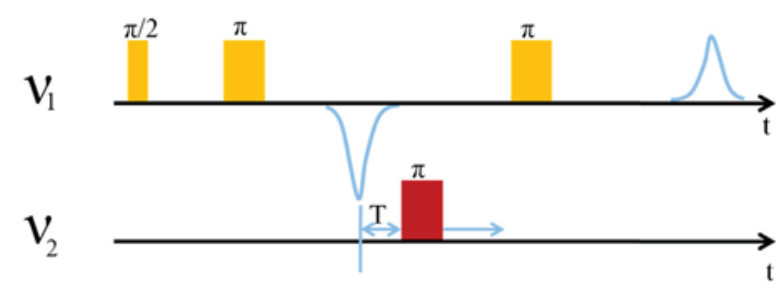

B

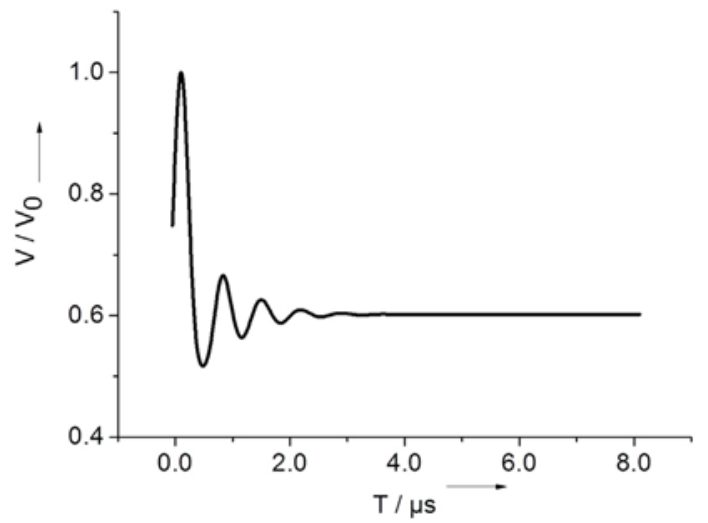

C

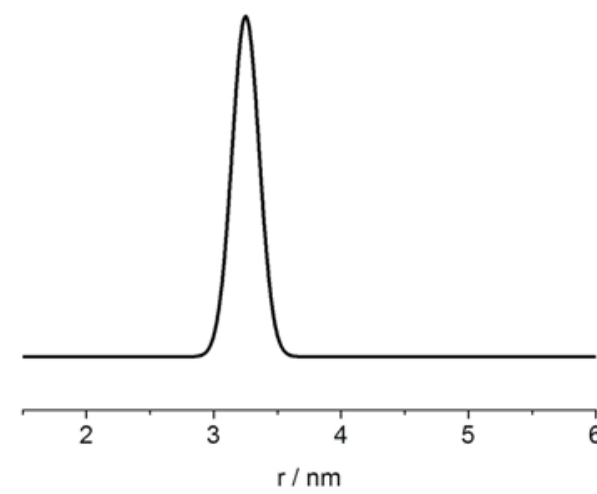

Figure 2. EPR distance measurements. (A) Pulse sequence of the dead-time free four-pulse DEER experiment. (B) Simulated DEER data for a doubly labeled model system. The intensity $V$ of the refocused observer echo (cf. A) is plotted versus the delay $T$ of the pump pulse (black). (C) Corresponding distance distribution. Adapted from .59

\section{Structure determination of DNA with EPR spectroscopy}

Structure elucidation of DNA sequences by electron paramag netic resonance (EPR) complements $\mathrm{X}$ ray crystallography and NMR spectroscopy, as it can be applied in noncrystalline states, is not intrinsically limited by the size of the bio macromolecular complex, and is able to analyze flexible structures or co existing DNA conformation. ${ }^{54}$ Since EPR can only be applied to species that contain unpaired electrons, the common approach exploits site directed spin labeling (SDLS) of DNA, usually with nitroxides as spin labels (see Section 3.2). ${ }^{55,56}$ Of particular interest are EPR tech niques giving access to long range distance constraints and distance distributions. Those distance measurements rely on the dipole dipole coupling between spin labels which is inversely pro portional to the cube of the distance. Pulsed EPR methods increase the range of distance sensitivity, double electron electron reso nance [DEER, an acronym which is synonymously used with PEL DOR (Pulsed Electron Double Resonance)] can access distances of up to $10 \mathrm{~nm}$ or more. . $^{57,58}$

For studying conformations of DNA oligonucleotides via EPR distance measurements usually two spin labels have to be introduced to determine their dipole dipole interaction via DEER measurements and to extract long range distance constraints. In contrast to Förster resonance energy transfer (FRET), which is widely used for studying distances in DNA, DEER does not require the use of two mutually distinct, relatively bulky chromophores, which have to be chosen depending on the expected distance. DEER distance measurements can be performed using two much smaller, identical spin labels, which are precise over a wide range of distances. The pulse sequence of the most widely used four pulse DEER experiment is shown in Figure 2A. ${ }^{57}$ We consider DNA molecules labeled with two chemically identical spin labels which feature different resonance frequencies $v_{1}$ and $v_{2}$, respec tively. Therefore, it is possible to apply pulses at two different fre quencies, the pulse sequence at $v_{1}$ addresses the so called $\mathrm{A}$ spins only, while the pulse at $v_{2}$ flips the B spins. At frequency $v_{1}$ a pulse sequence leading to a refocused echo of the spins A. Applying the pump pulse at the second frequency $v_{2}$ at variable time $T$ results in a modulation of the refocused echo intensity $V$ with the fre quency of the dipolar coupling between A and B spins. Plotting $V$ versus $T$ yields the typical DEER curve (Fig. $2 \mathrm{~B}$ ) which is used to ex tract the distance distribution between the spin labels (Fig. 2C).

For proof of concept experiments DNA based nanometer distance rulers were developed. ${ }^{60-62}$ Those are series of comple mentary oligonucleotides of which each bears one nitroxide spin label. Hybridization results in a double spin labeled DNA duplex. For different interspin distances molecular dynamics sim ulations showed very good correlation with DEER results. DNA dynamic measurements with DEER verified conformational flexi bility models. Series of double spin labeled DNA double helices were created (the separation between the two nitroxide spin labels was increased in 1 nt steps). The results supported a model for DNA stretching with a constant pitch. ${ }^{63}$

Recently, in cell DEER studies of DNA were developed. Com pared to in cell NMR spectroscopy, ${ }^{64}$ the novel approach of in cell electron paramagnetic resonance spectroscopy ${ }^{65}$ has two main advantages. Low concentrations can be used because EPR is much more sensitive per spin than NMR spectroscopy, and no back ground from diamagnetic molecules is observed. By now all in cell SDSL EPR studies on DNA have used oocytes from the African frog Xenopus laevis (X. laevis) which are widely used model systems in cellular and developmental biology. ${ }^{66-68}$ Spin labeled nucleic acids can be delivered into these cells via microinjection. A double spin labeled 7 bp long DNA ruler was delivered into X. laevis oocytes. In cellulo the distance measured between the labeled terminal thymi dines $(3.2 \mathrm{~nm})$ correlated to the theoretically calculated ones and was in accordance with those measured for the same double helix in vitro. Broadening of the in cell distance distribution indicated partial DNA melting. ${ }^{69}$ A 12 bp DNA double strand with higher relative content of $G C$ pairs, and thus higher stability than the 7 bp duplex displayed in cellulo the same conformation as it did in vitro. Furthermore, a second broader component of the distance distribution was assigned to intermolecular distances and inter preted as evidence of end to end stacking of the DNA duplexes and of duplex ordering into semi rigid rod like structures. ${ }^{66}$

\subsection{Structural elucidation of HTQ via DEER spectroscopy}

We have introduced EPR distance measurements for the inves tigation of highly polymorphic DNA quadruplex structures. ${ }^{67}$ While NMR spectroscopic and crystallographic studies have deliv ered impressive high resolution structures of human telomeric quadruplexes, biophysical studies indicate similar stabilities of these structures and provide increasing evidence of the co exis tence of some of these folds under physiological conditions. The high resolution methods were not able to decipher the exact nature of these structures under near physiological conditions 
since these techniques require the presence of single species. Due to the polymorphic nature of this DNA sequence, conducting DEER experiments on the human telomeric quadruplex is of particular interest. Studies of the HTQ formed by the sequence ((GGGTTA) ${ }_{3}$ GGG) suggested the coexistence of different conforma tions: (1) NMR data showed an anti parallel basket quadruplex topology in $\mathrm{Na}^{+}$containing solution; ${ }^{70}(2)$ crystals grown in the presence of $\mathrm{K}^{+}$depicted a parallel propeller form; ${ }^{71}$ (3) studies in $\mathrm{K}^{+}$containing solutions displayed controversial topologies. ${ }^{72-85}$

DEER spectroscopy allowed us to assign that the studied HTQ sequence adopts a 1:1 mixture of the parallel propeller and the anti parallel basket structure in $\mathrm{K}^{+}$solution (Fig. $3 \mathrm{~A} \mathrm{D}$ ) ${ }^{67}$ Even in cellulo the conformation of the HTQ was determined by DEER spectroscopy. For a usual measurement we inject $50 \mathrm{~nL}$ of a $4 \mathrm{mM}$ DNA solution into $X$. laevis oocytes (for a detailed protocol see Ref.65), resulting in a final intracellular concentration of $200 \mu \mathrm{M}$ assuming even distribution within the oocyte. Upon microinjection of the unfolded sequence into X. laevis oocytes the maxima of the distance distribution were assigned to the parallel propeller and the anti parallel basket conformations the same mixture as obtained in vitro. The existence of intermolecular quad ruplexes could be excluded. ${ }^{68}$ However, since the exact topology of the human telomeric sequence is also very much dependent on flanking nucleotides, it is important to study quadruplex confor mation of extended sequences as they better mimic the $3^{\prime}$ single stranded overhang of human telomeric DNA. EPR spectroscopy is very suited as technique for studying individual or local structures in larger contexts or complex media since only the spin labeled part is observed without the extended sequence contributing to background signals. Hence we studied the $G$ quadruplex confor mations within a sequence of three quadruplexes of human telo meric DNA by EPR spectroscopy. Here the formation of a $(3+1)$ hybrid topology was observed, contrary to the observation of the short, single HTQ quadruplexes described above (Fig. 3E G). ${ }^{87}$ Taken together, these studies confirmed that the human telomeric repeat can adopt different structures that are influenced by the cat ion present, the flanking nucleotides and the nucleic acid concen tration. ${ }^{88}$ Future experiments in cells should focus on the in vivo structure of extended human telomeric repeats. In addition possi ble interactions with biological factors present in cells should be investigated. With respect to the latter perspective, a potential lim itation needs to be considered: The amount of labeled DNA intro duced into the cells (resulting in approximately $200 \mu \mathrm{M}$ intracellular concentration) might be too high in order to observe interactions with factors present inside the cell at lower concentra tions. Hence, the development of even more sensitive labels
A

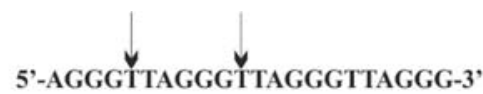

B



C antiparallel basket:



$1.7 \mathrm{~nm}$

5'-AG,TTAG ${ }_{3}$ TTAG,TTAG,TTAG,TTAG,TTAG,TTAG,TTAG,TTAG,TTAG,TTAG,-3'

$\mathbf{F}$

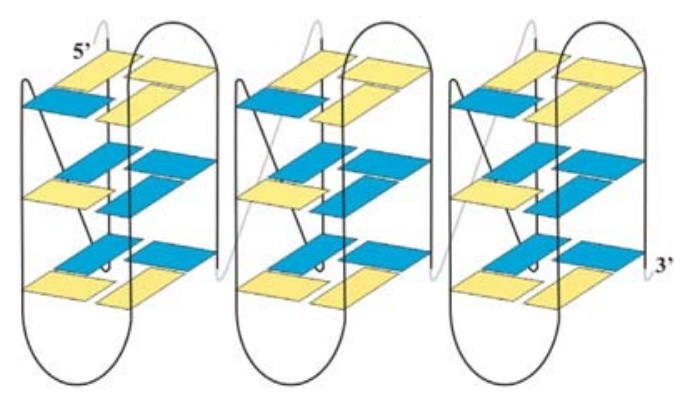

G

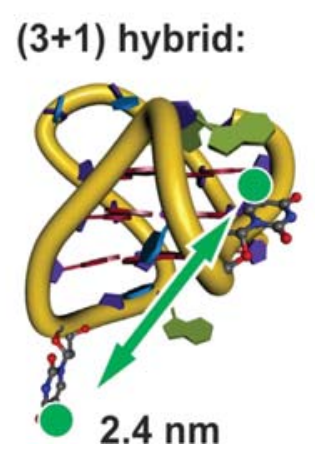

Figure 3. DEER measurements of HTQ. ${ }^{67}$ (A) Sequence of a single HTQ. Arrows indicate sites of 5-TPA-modified deoxyuridine (for details of the spin-label see Fig. 4E). (B) Distance distributions of DEER spectroscopy data for the A(GGGTTA) ${ }_{3}$ GGG sequence in the presence of $\mathrm{K}^{+}$. Two distinct species are observed: anti-parallel basket (red arrow) and parallel propeller (blue arrow) (C, D and G) Different intramolecular quadruplex topologies based on high resolution structures. Arrows indicate distances measured between spin-labeled C5 methyl carbon atoms of deoxythymidine residues on the basis of the PDB structures. (C) NMR spectroscopy structure of the anti-parallel basket quadruplex in $\mathrm{Na}^{+}$ion containing solution (PDB: 143D). ${ }^{70}$ (D) Crystal structure of the parallel propeller form in the presence of $\mathrm{K}^{+}$ions (PDB: $\left.1 \mathrm{KF} 1\right)^{71}$ (G) NMR spectroscopic structure of a (3+1) hybrid quadruplex of a slightly modified sequence in $\mathrm{K}^{+}$ions (PDB: $2 \mathrm{GKU}$ ). ${ }^{86}$ (E) Sequence of the investigated elongated HTQ (the middle and $5^{\prime}$-terminal quadruplex units were labeled as depicted in A). (F) Scheme for the (3+1) hybrid structured elongated HTQ. 
allowing to further decrease the intracellular sample concentration is of general interest.

\subsection{Site directed spin-labeling of DNA}

To monitor the structure and dynamics of DNA via EPR spec troscopy paramagnetic centers in the macromolecule are required. Incorporation of spin labels in a nucleotide sequence is enabled by a site directed spin labeling approach (SDSL). ${ }^{89}$ Thereby DNA se quences without native paramagnetic centers can be investigated. The most common spin labels for SDSL EPR are nitroxides with the general formula $O N R_{1} R_{2}$ (Fig. 4A). They are chemically inert, sta ble radicals with the unpaired electron delocalized on the $\mathrm{N} \mathrm{O}$ bond. The stability of the nitroxides is highly dependent on their structure: variations near the $\mathrm{N} \mathrm{O}$ bond have the greatest influ ence. The sticking point is a disproportionation reaction, in which the nitroxide is reduced to the corresponding amine. ${ }^{90}$ Especially in cells the half life of nitroxide spin labels is short. Michaelis Menten behavior of label quenching suggests additional enzymatic reduction processes in cellular environments. ${ }^{91}$ Embedding the nitrogen in a heterocyclic ring can enhance its stability: five and six membered ring nitroxides are commonly used. Several differ ent spin labels, TEMPO like (2,2,6,6 tetramethylpiperidine 1 oxyl), TPA (2,2,5,5 tetramethyl pyrrolin 1 oxyl 3 acetylene) or nitrox ide bearing isoindole moieties have been reported among others (Fig. 4B, D and C). ${ }^{92}$ It was shown that five membered rings have higher intracellular stability than six membered ring nitroxides. ${ }^{91}$

Specific functional groups are used for coupling of nitroxides to nucleotides. In principle nucleic acids can be labeled at many posi tions such as the phosphodiester group ${ }^{93}$, the sugar moiety ${ }^{94}$ or the nucleobase. The purines adenine and guanine can be labeled at the amino group ( 6 and 2 position). ${ }^{95}$ Also, guanines bearing a nitroxide in the 06 position ${ }^{96}$ and adenines carrying a spin label at the 2 position via an acetylenic tether ${ }^{97}$ have been reported. Pyrimidine nucleobases can be used for spin labeling as well. In cytosine the spin label was attached to the 4 position $^{98}$ and for thymine two types of spin labeled analogues have been reported: (1) the nitroxide attached by a flexible linker to the sulfur atom of a 4 thiosubstituated deoxyuridine,,$^{99}(2)$ the nitroxide attached to a 5 iodo 2 ' deoxyuridine via an acetylenic tether (Fig. 3E). ${ }^{100,101}$ The latter is performed via a Sonogashira cross cou pling reaction in solution. The nucleoside analogue is then con verted to the labeled phosphoramidite. ${ }^{102}$ This spin label strategy is frequently utilized in EPR spectroscopy of nucleic acids. The nitr oxide in the 5 position points into the major groove of the DNA and does not disturb duplex DNA, as determined by CD spectros copy. ${ }^{103}$ Spin labeled phosphoramidites can be inserted at desired positions in oligonucleotides using automated DNA synthesis. The

A

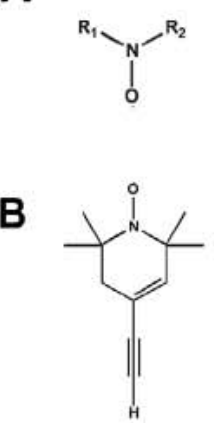

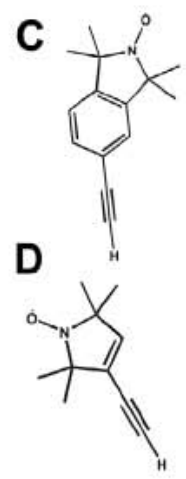



Figure 4. Commonly used nitroxide spin labels. (A) General nitroxide structure. (B) TEMPO-like spin-label. (C) Isoindole-like spin-label. (D) TPA-like spin-label. (D) TPA spin-label attached to uridine via acetylenic tether. nitroxide can be either introduced during solid phase DNA synthe sis by direct coupling of the labeled phosphoramidite ${ }^{102}$ or the Sonogashira cross coupling is performed on the CPG solid support after incorporation of 5 iodo deoxyuridine. ${ }^{92}$ In principle labeled sequences longer than $100 \mathrm{nt}$ can be synthesized automatically but each round of coupling reduces a proportion of the nitroxide to the EPR silent hydroxylamine. Changing the standard iodine based oxidizer with tert butyl hydroperoxide has shown to de crease the amount of nitroxide to hydroxylamine reduction. ${ }^{90}$ Problems during the solid phase synthesis might be overcome by enzymatic synthesis: a sequence with eleven adjacent spin labels was generated by a DNA polymerase catalyzed incorporation reac tion of spin labelled nucleoside triphosphates. ${ }^{104}$ Also post synthetic DNA functionalization via click chemistry ${ }^{105}$ or targeting a modified phosphodiester group is possible. ${ }^{61}$ The final choice of the spin labeling strategy depends on the sample probe and the addressed problem.

\section{Conclusions}

Structural characterization of noncanonical DNA conformations such as the human telomeric quadruplex in living cells is an inter esting and emerging field. Since EPR relies on the observation of a paramagnetic center and such stable unpaired radicals are rarely found inside cells, EPR with a low background noise is a very suitable technique for measuring in a biological medium after microinjecting or transfecting the spin labeled DNA. Future aims are the development of even more stable spin labels with increased rigidity. In addition, increasing the EPR sensitivity might be feasi ble and delivery strategies apart from injection into frog oocytes should be explored. In the DEER experiments on HTQ discussed in more detail in this perspective the spin labels were attached to the thymine bases, located in the quadruplex loop. Since the loops possess intrinsically high flexibility broad distance distribu tions are obtained in the EPR data. Hence it might be beneficial to attach spin labels at structurally more defined positions such as the guanines itself. However, as with all labeling approaches one has to take care that the attachment of the label itself is not influencing the structure and dynamics of the studied system.

\section{Acknowledgments}

J.S.H. acknowledges the Volkswagen Stiftung for funding a Lichtenberg Professorship. This work was financially supported by the DFG (DR 743/2 1). We thank Daniel Dietrich and Oliver Okle as well as all members of the Drescher and Hartig groups who con tributed to this topic, especially Mykhailo Azarkh and Vijay Singh.

\section{References and notes}

1. Watson, J. D.; Crick, F. H. Nature 1953, 171, 737.

2. Bochman, M. L; Paeschke, K.; Zakian, V. A. Nat. Rev. Genet, 2012, 13, 770

3. Pearson, C. E.; Zorbas, H.; Price, G. B.; Zannis-Hadjopoulos, M. J. Cell. Biochem. $1996,63,1$.

4. Singleton, M. R.; Scaife, S.; Raven, N. D.; Wigley, D. B. Acta Crystallogr., Sect. D 2001, 57, 1695.

5. Yuan, C; Rhoades, E.; Heuer, D. M.; Archer, L. A. Biophys. J. 2005, 89, 2564.

6. Lobachev, K. S.; Rattray, A.; Narayanan, V. Front. Biosci. 2007, 12, 4208.

7. Palecek, E. Crit. Rev. Biochem. Mol. Biol. 1991, 26, 151.

8. Hoyne, P. R.; Edwards, L. M.; Viari, A.; Maher, L. J., 3rd. J. Mol. Biol. 2000, 302, 797

9. Hoyne, P. R.; Gacy, A. M.; McMurray, C. T.; Maher, L. J., 3rd. Nucleic Acids Res. $2000,28,770$.

10. Jain, A.; Wang, G.; Vasquez, K. M. Biochimie 2008, 90, 1117.

11. Capra, J. A.; Paeschke, K.; Singh, M.; Zakian, V. A. PLoS Comput. Biol. 2010, 6, e1000861.

12. Rawal, P.; Kummarasetti, V. B.; Ravindran, J.; Kumar, N.; Halder, K.; Sharma, R.; Mukerji, M.; Das, S. K.; Chowdhury, S. Genome Res. 2006, 16, 644.

13. Hershman, S. G.; Chen, Q.; Lee, J. Y.; Kozak, M. L.; Yue, P.; Wang, L. S.; Johnson, F. B. Nucleic Acids Res. 2008, 36, 144. 
14. Paramasivan, S.; Rujan, I.; Bolton, P. H. Methods 2007, 43, 324

15. Kypr, J.; Kejnovska, I.; Renciuk, D.; Vorlickova, M. Nucleic Acids Res. 2009, 37. 1713.

16. Campbell, N. H.; Parkinson, G. N. Methods 2007, 43, 252

17. Webba da Silva, M. Methods 2007, 43, 264.

18. Gellert, M.; Lipsett, M. N.; Davies, D. R. Proc. Natl. Acad. Sci. U.S.A. 2013, 1962, 48.

19. Joachimi, A.; Benz, A.; Hartig, J. S. Bioorg. Med. Chem. 2009, 17, 6811.

20. Burge, S.; Parkinson, G. N.; Hazel, P.; Todd, A. K.; Neidle, S. Nucleic Acids Res. 2006, 34, 5402.

21. Halder, R.; Riou, J. F.; Teulade-Fichou, M. P.; Frickey, T.; Hartig, J. S. BMC Res. Notes 2012, 5, 138 .

22. Alberti, P.; Ren, J.; Teulade-Fichou, M. P.; Guittat, L.; Riou, J. F.; Chaires, J.; Helene, C.; Vigneron, J. P.; Lehn, J. M.; Mergny, J. L. J. Biomol. Struct. Dyn. 2001, 19, 505.

23. Nielsen, M. C.; Ulven, T. Curr. Med. Chem. 2010, 17, 3438.

24. Wong, H. M.; Payet, L.; Huppert, J. L. Curr. Opin. Mol. Ther. 2009, 11, 146.

25. Todd, A. K.; Johnston, M.; Neidle, S. Nucleic Acids Res. 2005, 33, 2901

26. Huppert, J. L.; Balasubramanian, S. Nucleic Acids Res. 2005, 33, 2908.

27. Eddy, J.; Maizels, N. Nucleic Acids Res. 2006, 34, 3887.

28. Beaume, N.; Pathak, R.; Yadav, V. K.; Kota, S.; Misra, H. S.; Gautam, H. K.; Chowdhury, S. Nucleic Acids Res. 2013, 41, 76.

29. Nakken, S.; Rognes, T.; Hovig, E. Nucleic Acids Res. 2009, 37, 5749.

30. Sun, D.; Hurley, L. H. J. Med. Chem. 2009, 52, 2863.

31. Brooks, T. A.; Kendrick, S.; Hurley, L. FEBS J. 2010, 277, 3459.

32. Paeschke, K.; Capra, J. A.; Zakian, V. A. Cell 2011, 145, 678.

33. Lopes, J.; Piazza, A.; Bermejo, R.; Kriegsman, B.; Colosio, A.; Teulade-Fichou, M. P.; Foiani, M.; Nicolas, A. EMBO J. 2011, 30, 4033.

34. Sarkies, P.; Reams, C.; Simpson, L. J.; Sale, J. E. Mol. Cell 2010, 40, 703.

35. Cahoon, L. A.; Seifert, H. S. Science 2009, 325, 764.

36. Schaffitzel, C.; Pluckthun, A. Trends Biochem. Sci. 2001, 26, 577.

37. Yang, Q.; Xiang, J.; Yang, S.; Zhou, Q.; Li, Q.; Tang, Y.; Xu, G. Chem. Commun. 2009, 1103.

38. Chang, C. C.; Kuo, I. C.; Lin, J. J.; Lu, Y. C.; Chen, C. T.; Back, H. T.; Lou, P. J.; Chang, T. C. Chem. Biodiv. 2004, 1, 1377.

39. Biffi, G.; Tannahill, D.; McCafferty, J.; Balasubramanian, S. Nat. Chem. 2013, 5, 182.

40. Makarov, V. L.; Hirose, Y.; Langmore, J. P. Cell 1997, 88, 657.

41. McElligott, R.; Wellinger, R. J. EMBO J. 1997, 16, 3705.

42. Harley, C. B.; Futcher, A. B.; Greider, C. W. Nature 1990, 345, 458.

43. Sfeir, A. J.; Chai, W.; Shay, J. W.; Wright, W. E. Mol. Cell 2005, 18, 131

44. Healy, K. C. Oncol. Res. 1995, 7, 121.

45. Zahler, A. M.; Williamson, J. R.; Cech, T. R.; Prescott, D. M. Nature 1991, 350, 718.

46. Oganesian, L.; Moon, I. K.; Bryan, T. M.; Jarstfer, M. B. EMBO J. 2006, 25, 1148.

47. Oganesian, L.; Graham, M. E.; Robinson, P. J.; Bryan, T. M. Biochemistry 2007, $46,11279$.

48. Shay, J. W.; Wright, W. E. Semin. Cancer Biol. 2011, 21, 349.

49. Hurley, L. H. Nat. Rev. Cancer 2002, 2, 188.

50. Neidle, S.; Parkinson, G. Nat. Rev. Drug Discovery 2002, 1, 383.

51. Harley, C. B. Mutat. Res. 1991, 256, 271

52. Bodnar, A. G.; Ouellette, M.; Frolkis, M.; Holt, S. E.; Chiu, C. P.; Morin, G. B.; Harley, C. B.; Shay, J. W.; Lichtsteiner, S.; Wright, W. E. Science 1998, 279, 349.

53. Hackett, J. A.; Feldser, D. M.; Greider, C. W. Cell 2001, 106, 275.

54. Drescher, M.; Jeschke, G., Eds. In EPR Spectroscopy: Applications in Chemistry and Biology. Topics in Current Chemistry. Springer Verlag Berlin Heidelberg, 2012. 321, 1 .

55. Likhtenshtein, G.; Yamauchi, J.; Nakatsuji, S.; Smirnov, A.; Tamura, R. Nitroxides; Wiley-VCH, Weinheim, 2008; Vol. XIX, 419 p.

56. Berliner, L.; Reuben, J. Biol. Magn. Reson. 1989, 8, 1.

57. Pannier, M.; Veit, S.; Godt, A.; Jeschke, G.; Spiess, H. W. J. Magn. Reson. 2000, 142, 331 .

58. Ward, R.; Bowman, A.; Sozudogru, E.; El-Mkami, H.; Owen-Hughes, T.; Norman, D. G. J. Magn. Reson. 2010, 207, 164.

59. Drescher, M.; Jeschke, G. Top. Curr. Chem. 2012, 321.

60. Schiemann, O.; Piton, N.; Mu, Y.; Stock, G.; Engels, J. W.; Prisner, T. F. J. Am. Chem. Soc. 2004, 126, 5722

61. Cai, Q: Kusnetzow, A. K.; Hubbell, W. L.; Haworth, I. S.; Gacho, G. P.; Van Eps, N.; Hideg, K.; Chambers, E. J.; Qin, P. Z. Nucleic Acids Res. 2006, 34, 4722.

62. Ward, R.; Keeble, D. J.; El-Mkami, H.; Norman, D. G. ChemBioChem 1957, 2007, 8.
63. Marko, A.; Denysenkov, V.; Margraf, D.; Cekan, P.; Schiemann, O.; Sigurdsson, S. T. Prisner, T. F. J. Am. Chem. Soc. 2011, 133, 13375.

64. Serber, Z.; Selenko, P.; Hansel, R.; Reckel, S.; Lohr, F.; Ferrell, J. E., Jr.; Wagner, G.; Dotsch, V. Nat. Protocols 2006, 1, 2701.

65. Azarkh, M.; Singh, V.; Okle, O.; Seemann, I. T.; Dietrich, D. R.; Hartig, J. S.; Drescher, M. Nat. Protocols 2013, 8, 131.

66. Krstic, I.; Hansel, R.; Romainczyk, O.; Engels, J. W.; Dotsch, V.; Prisner, T. F. Angew. Chem. 2011, 50, 5070.

67. Singh, V.; Azarkh, M.; Exner, T. E.; Hartig, J. S.; Drescher, M. Angew. Chem. 2009, 48, 9728

68. Azarkh, M.; Singh, V.; Okle, O.; Dietrich, D. R.; Hartig, J. S.; Drescher, M. ChemPhysChem 2012, 13, 1444

69. Azarkh, M.; Okle, O.; Singh, V.; Seemann, I. T.; Hartig, J. S.; Dietrich, D. R.; Drescher, M. ChemBioChem 1992, 2011, 12.

70. Wang, Y.; Patel, D. J. Structure 1993, 1, 263.

71. Parkinson, G. N.; Lee, M. P.; Neidle, S. Nature 2002, 417, 876

72. Dai, J.; Carver, M.; Yang, D. Biochimie 2008, 90, 1172.

73. Ambrus, A.; Chen, D.; Dai, J.; Bialis, T.; Jones, R. A.; Yang, D. Nucleic Acids Res. 2006, 34, 2723.

74. He, Y.; Neumann, R. D.; Panyutin, I. G. Nucleic Acids Res. 2004, 32, 5359

75. Ying, L.; Green, J. J.; Li, H.; Klenerman, D.; Balasubramanian, S. Proc. Natl. Acad. Sci. U.S.A. 2003, 100, 14629

76. Risitano, A.; Fox, K. R. Bioorg. Med. Chem. Lett. 2005, 15, 2047

77. Rujan, I. N.; Meleney, J. C.; Bolton, P. H. Nucleic Acids Res. 2005, 33, 2022.

78. Ourliac-Garnier, I.; Elizondo-Riojas, M. A.; Redon, S.; Farrell, N. P.; Bombard, S. Biochemistry 2005, 44, 10620.

79. Wlodarczyk, A.; Grzybowski, P.; Patkowski, A.; Dobek, A. J. Phys. Chem. B 2005, 109, 3594.

80. Qi, J.; Shafer, R. H. Nucleic Acids Res. 2005, 33, 3185.

81. Redon, S.; Bombard, S.; Elizondo-Riojas, M. A.; Chottard, J. C. Nucleic Acids Res. 2003, 31, 1605

82. Li, J.; Correia, J. J.; Wang, L.; Trent, J. O.; Chaires, J. B. Nucleic Acids Res. 2005, 33, 4649.

83. Risitano, A.; Fox, K. R. Nucleic Acids Res. 2004, 32, 2598.

84. Hazel, P.; Huppert, J.; Balasubramanian, S.; Neidle, S. J. Am. Chem. Soc. 2004, 126,16405

85. Benz, A.; Hartig, J. S. Chem. Commun. 2008, 4010.

86. Phan, A. T.; Kuryavyi, V.; Luu, K. N.; Patel, D. J. Nucleic Acids Res. 2007, 35, 6517.

87. Singh, V.; Azarkh, M.; Drescher, M.; Hartig, J. S. Chem. Commun. 2012, 48, 8258.

88. Neidle, S. Curr. Opin. Struct. Biol. 2009, 19, 239.

89. Hubbell, W. L.; Cafiso, D. S.; Altenbach, C. Nat. Struct. Biol. 2000, 7, 735.

90. Cekan, P.; Smith, A. L.; Barhate, N.; Robinson, B. H.; Sigurdsson, S. T. Nucleic Acids Res. 2008, 36, 5946

91. Azarkh, M.; Okle, O.; Eyring, P.; Dietrich, D. R.; Drescher, M. J. Magn. Reson. 2011, 212, 450.

92. Schiemann, O.; Piton, N.; Plackmeyer, J.; Bode, B. E.; Prisner, T. F.; Engels, J. W. Nat. Protocols 2007, 2, 904

93. Qin, P. Z.; Butcher, S. E.; Feigon, J.; Hubbell, W. L. Biochemistry 2001, 40, 6929.

94. Edwards, T. E.; Okonogi, T. M.; Robinson, B. H.; Sigurdsson, S. T. J. Am. Chem. Soc. 2001, 123, 1527.

95. Sicoli, G.; Wachowius, F.; Bennati, M.; Hobartner, C. Angew. Chem. 2010, 49, 6443.

96. Giordano, C.; Pedone, F.; Fattibene, P.; Cellai, L. Nucleosides, Nucleotides and Nucleic acids 2000, 19, 1301.

97. Piton, N.; Mu, Y.; Stock, G.; Prisner, T. F.; Schiemann, O.; Engels, J. W. Nucleic Acids Res. 2007, 35, 3128.

98. Cekan, P.; Sigurdsson, S. T. J. Am. Chem. Soc. 2009, 131, 18054.

99. Qin, P. Z.; Hideg, K.; Feigon, J.; Hubbell, W. L. Biochemistry 2003, 42, 6772.

100. Spaltenstein, A.; Robinson, B. H.; Hopkins, P. B. Biochemistry 1989, 28, 9484.

101. Gannett, P. M.; Darian, E.; Powell, J.; Johnson, E. M., 2nd; Mundoma, C. Greenbaum, N. L.; Ramsey, C. M.; Dalal, N. S.; Budil, D. E. Nucleic Acids Res. 2002, 30, 5328.

102. Azarkh, M.; Singh, V.; Okle, O.; Seemann, I. T.; Dietrich, D.; Hartig, J. S.; Drescher, M. Nat. Protoc. 2013, 8, 131.

103. Hustedt, E. J.; Kirchner, J. J.; Spaltenstein, A.; Hopkins, P. B.; Robinson, B. H. Biochemistry 1995, 34, 4369.

104. Obeid, S.; Yulikov, M.; Jeschke, G.; Marx, A. Angew. Chem. 2008, 47, 6782

105. Jakobsen, U.; Shelke, S. A.; Vogel, S.; Sigurdsson, S. T. J. Am. Chem. Soc. 2010 , $132,10424$. 